\title{
BMJ Open Cognitive training and neuroplasticity in mild cognitive impairment (COG-IT): protocol for a two-site, blinded, randomised, controlled treatment trial
}

\author{
Jessica D'Antonio, ${ }^{1}$ Laura Simon-Pearson, ${ }^{\circ} 1$ Terry Goldberg, ${ }^{1,2}$ Joel R Sneed, ${ }^{1,3}$ \\ Sara Rushia, ${ }^{3,4}$ Nancy Kerner, ${ }^{2}$ Howard Andrews, ${ }^{5}$ Caroline Hellegers, ${ }^{6}$ \\ Sierra Tolbert, ${ }^{6}$ Elena Perea, ${ }^{6}$ Jeffrey Petrella, ${ }^{7}$ Murali Doraiswamy, ${ }^{6}$ \\ Davangere Devanand ${ }^{1,2}$
}

To cite: D'Antonio J, SimonPearson L, Goldberg T, et al. Cognitive training and neuroplasticity in mild cognitive impairment (COGIT): protocol for a two-site, blinded, randomised, controlled treatment trial. BMJ Open 2019;9:e028536. doi:10.1136/ bmjopen-2018-028536

- Prepublication history for this paper is available online. To view these files, please visit the journal online (http://dx.doi org/10.1136/bmjopen-2018028536).

Received 20 December 2018 Revised 17 June 2019 Accepted 10 July 2019

Check for updates

(C) Author(s) (or their employer(s)) 2019. Re-use permitted under CC BY-NC. No commercial re-use. See rights and permissions. Published by BMJ.

For numbered affiliations see end of article.

Correspondence to Dr Davangere Devanand; dpd3@cumc.columbia.edu

\section{ABSTRACT}

Introduction Mild cognitive impairment $(\mathrm{MCl})$ is common in older adults and represents a high-risk group for progression to Alzheimer's disease (AD). Medication trials in $\mathrm{MCl}$ have generally failed, but new discoveries with brain plasticity in ageing have led to the study of cognitive training as a potential treatment to improve cognitive abilities. Computerised cognitive training (CCT) involves computerised cognitive exercises that target specific cognitive abilities and neural networks to potentially improve cognitive functioning through neuroplasticity.

Methods and analysis In a two-site study (New York State Psychiatric Institute/Columbia University Medical Center and Duke University Medical Center), we will randomise 100 patients with $\mathrm{MCl}$ (Wechsler Memory Scale-III Logical Memory II score 0-11; Folstein Mini Mental State Examination $\geq 23$ ) to home-based CCT (suite of exercises: memory, matching, spatial recognition, processing speed) or a home-based active control condition (computerised crossword puzzle training (CPT)) with 12 weeks of intensive training followed by regular booster sessions up to 78 weeks. All patients will receive standard neuropsychological and functional assessments in clinic as well as structural/functional brain MRI scans at study entry and endpoint. We will test if CCT, versus $\mathrm{CPT}$, leads to improved cognitive functioning, transfers to functional ability and tasks of everyday life and impacts hippocampal volume changes and changes in the default mode network of the brain measured by resting-state functional MRI.

Ethics and dissemination The study will be conducted following ethics approval and written informed consent will be obtained from all subjects. Study results will be disseminated via publication, clinicaltrials.gov, media and conference presentations. This will be the first controlled long-term trial to evaluate the effects of home-based CCT versus computerised CPT on cognitive abilities and functional measures and neural outcomes as determined by MRI indices in patients with $\mathrm{MCl}$. Positive results from trial may support further development of home-based CCT. Trial registration number ClinicalTrials.gov identifier (NCT03205709).

\section{Strengths and limitations of this study}

- The study will improve on limitations of most previous studies by including an 'active' control condition rather than waitlist or control conditions that do not account for engagement and motivation.

- This study will evaluate performance on traditional cognitive and functional assessments (eg, Alzheimer's Disease Assessment Scale-Cognition Subscale 11, University of California San Diego Performance-Based Skills Assessment) in addition to performance on a self-administered, computerised cognitive test, the NeuroCognitive Performance Test, which consists of 10 subtests that are online adaptations of widely used neuropsychological tests.

- The trial will use a remote Internet-based computerised cognitive training (CCT) intervention that can be done at home; compared with most existing treatments under investigation, it is easily accessible, relatively inexpensive, non-invasive and scaled to the skill level of each individual.

- The trial will include evaluation of clinically relevant genetic, brain network and neuronal loss markers as moderators of outcome; this will be one of the first trials to examine long-term effects on cognition, daily functioning and neuroplastic changes in default mode network with CCT in mild cognitive impairment.

- As our trial will be restricted to English-speaking participants because the online training platform is only available in English, we are unsure how this will generalise to non-English speaking individuals. In addition, the inclusion criteria state that the participant must have an at-home desktop or laptop computer, which, in low socioeconomic class homes, is not always available.

\section{INTRODUCTION}

Alzheimer's disease (AD) is a major public health concern affecting over 40 million people worldwide, and there is an urgent need to develop new treatment modalities 
to prevent or delay the onset of dementia. Mild cognitive impairment (MCI) is common in older adults and represents a high-risk group for $\mathrm{AD}$, but medication trials in MCI have generally failed. There is no Food and Drug Administration-approved treatment for MCI or to prevent the progression of $\mathrm{MCI}$ to $\mathrm{AD}$.

There is growing evidence that a cognitively active lifestyle may reduce rates of dementia. A systematic review of 22 population-based studies found that mental activities may reduce overall incident dementia risk by $46 \%$ over a median 7 -year period. ${ }^{1}$ Computerised cognitive training (CCT) provides a novel strategy to improve cognitive performance in MCI by establishing a more cognitively active lifestyle. CCT involves computerised cognitive exercises that target specific abilities to improve cognitive functioning, and this effect is likely to be mediated by neuroplasticity. CCT has been used successfully to improve cognitive functioning in healthy adult populations. ${ }^{2-5}$ In particular, two studies in older healthy controls have garnered much attention. The Advanced Cognitive Training for Independent and Vital Elderly (ACTIVE) trial was the first large-scale, randomised trial to show evidence of transfer from cognitive training to improved daily function, but only in participants who completed reasoning or speed of processing focused training and not memory training. ${ }^{6}$ In this 10 -year follow-up study of 2,832 healthy adults (average age $=73.6$ ), participants were randomised to one of three intervention groups (memory, reasoning or speed of processing) or a no-contact control group. Booster sessions occurred for $39 \%$ of participants in all active groups at 11 and 35 months after initial training, but the cognitive effects of the memory training did not persist over the 10-year follow-up period. ${ }^{6}$ In a second large, well-publicised online cognitive training study, findings were originally considered to be negative in terms of cognitive gains. However, in an examination of an older subsample, training was effective in improving cognitive abilities and instrumental activities of daily living. ${ }^{7}$

In a recent meta-analysis examining CCT in older adults with MCI or dementia, the overall efficacy of cognitive outcomes in MCI was moderate and statistically significant. ${ }^{8}$ This pattern was also found for global cognition, verbal learning and memory, non-verbal learning, working memory, attention and psychosocial functioning (eg, depression, quality of life, neuropsychiatric symptoms). However, for the efficacy of cognitive outcomes in patients with dementia, the overall effect was found to be small, though statistically significant.

Early interventions at the stage of MCI, and not dementia, may be more helpful for improving cognition. In fact, Hill et $a l^{8}$ concluded that CCT is a feasible intervention for improving cognition in patients with MCI. Transfer effects have also been found in studies evaluating CCT in healthy older adults, supporting the potential for transfer of CCT benefits to daily life ${ }^{69}$ In this study, we will assess for transfer effects by administering the following functional assessments at specific timepoints:
Functional Activities Questionnaire (FAQ) and University of California San Diego Performance-Based Skills Assessment (UPSA).

Although CCT has received more support in the past few years as a viable treatment option for older adults with MCI, the brain mechanisms underlying the observed cognitive changes remain elusive. Many studies of CCT that include imaging components have only been conducted with healthy older adults. ${ }^{10-13}$ CCT may promote neuroplasticity in the brain, including in the hippocampus, a key region that supports memory. ${ }^{13-16}$ Additional research needs to be done that evaluates both structural and functional data within a rigorously conducted clinical trial. In this study, patients will undergo a structural MRI and functional MRI (fMRI) at both study entry and exit to assess for changes in hippocampal volume and the default mode network (DMN). The latter is crucial to evaluate in patients with MCI as dysfunction in the DMN has been implicated in the progression of MCI to $\mathrm{AD} .{ }^{17}$ The DMN is a resting state neural network of several highly interconnected cortical hubs, including the posteromedial parietal, anteromedial frontal and inferolateral parietal cortices. We have shown that impaired deactivation and functional connectivity in the DMN may be a significant predictor in MCI of poor memory and transition to dementia over a 2-3 year follow-up period. ${ }^{18}$ Neuronal dysfunction precedes structural atrophy in $\mathrm{AD}$, and fMRI offers the potential for identifying specific patterns of disruption in the memory networks affected early in MCI and AD.

Limitations of prior CCT trials include the inconsistent demonstration of transfer to everyday functioning, reliance on waitlist control conditions as opposed to active control conditions and lack of long-term follow-up. Most studies have not assessed transfer of cognitive improvement to everyday function or quality of life. ${ }^{19-23}$ While CCT may produce transfers to untrained cognitive domains, the few studies that evaluate transfer to everyday functioning have reported mixed findings. ${ }^{24-29}$ This is particularly important given the strong association between cognitive decline and functional disability. ${ }^{30}$ Many studies use waitlist control conditions or control conditions that do not account for engagement and motivation in the task. ${ }^{22}$ Such designs are biassed in favour of the treatment condition because waitlisted subjects are not receiving any form of cognitive treatment and, therefore, may be more likely to drop out of such studies due to lack of engagement and motivation. In the current study, patients will be assigned to one of two cognitively stimulating exercises, CCT (suite of exercises) or crossword puzzle training (CPT; crossword puzzles). Since one of the purposes of CCT in patients with MCI is to reduce the risk of progression to dementia, longer follow-up times are necessary to be able to accurately capture patient progression. However, most studies have only used no follow-up or short-term follow-ups, with the notable exception of the ACTIVE trial. ${ }^{631-33}$

Overall, recent findings in the field suggest that CCT could benefit patients at risk for dementia. The current 
study will build on these findings by implementing a study design with an active control group, a longer trial duration, an increased intensity of CCT, examination of generalisability to functional abilities beyond cognitive training skills, structural and fMRI assessment and rigorously blinded methodology.

\section{METHODS AND ANALYSIS}

\section{Study design features and rationale}

One hundred patients clinically diagnosed with MCI will be randomised. There will be two sites: New York State Psychiatric Institute/Columbia University Medical Center (CUMC) in New York, NY (NYSPI as lead coordinating site) and Duke University Medical Center (DUMC) in Durham, NC. Patients will be randomised to one of two computerised cognitively stimulating exercises: crossword puzzle training (CPT) or a suite of exercises (CCT; memory tasks, matching tasks, spatial recognition tasks, processing speed tasks). These patients will be further randomised by MCI type (early MCI or late MCI), age (70 and below or 71 and above) and site (NYSPI/CUMC or DUMC) as the stratification factors and will be followed for 78 weeks. The randomisation sequences will be balanced in blocks of random size $(2,4)$ to prevent clinicians from guessing what the next patient's treatment might be. The term 'control' will not be used in the consent form to reduce the participant's expectancy bias.

To maintain neutrality and mitigate expectancy bias among patients, the informed consent form signed by all patients during the screening visit will not indicate which group is the active group (suite of exercises) or the control group (crosswords). Rather, it will indicate that the patient may be assigned to one of two cognitively stimulating exercises, CCT or CPT.

\section{Role of sponsor}

The study is funded entirely by the National Institute on Aging grant and supervised by a Data Safety Monitoring Board. Using Lumosity, a web-based gaming platform from Lumos Labs, we customised a specific set of CCT and CPT training modules for participants to use in this trial. After a comprehensive review of several CCT modules on the market, we chose these modules from Lumosity due to their large selection of games tailored to specific cognitive domains, their research specific platform, availability of active control condition, availability of the online self-administered NeuroCognitive Performance Test (NCPT) and our previous pilot data in the elderly with this platform. Aside from providing the research platform and technical support at no cost, Lumos Labs provides no financial support for this study and their staff have no significant role in the final study design, study conduct, data interpretation or publication. Patients will not be required to pay for the platform and will not have a poststudy commitment to the platform. None of the study team has any financial conflicts with Lumos Labs.

\section{Recruitment, eligibility, consent}

Patients will be recruited from the current patient caseload of the investigators, referral by neurology, psychiatry, primary care, public health and geriatric medical clinics affiliated with the centres and supplemented by advertisement.

\section{Inclusion/exclusion criteria}

Detailed inclusion/exclusion criteria are described in box 1. Notable inclusion criteria will be age range restriction 55-95 years, subjective cognitive complaints (ie, memory or other cognitive complaints, eg, naming/language), Wechsler Memory Scale-III (WMS-III) Logical Memory Story A delayed recall score 0-11, Folstein Mini Mental State Examination (MMSE) score $\geq 23$ out of 30 , availability of an informant and access to a home desktop or laptop computer with full access to the Internet for the study duration. Patients who have a history of major psychiatric or neurological illness including motor disorders like Parkinson's disease, a dementia diagnosis of any type, contraindication to MRI scan, lack of English-speaking ability or have been defined as regular online brain training or regular crossword puzzle users ( $\geq 2$ times per week in the past year) will be excluded. Depression will be assessed using the 15-item Geriatric Depression Scale (GDS); a diagnosis of major depressive disorder is exclusionary.

\section{$\mathrm{MCl}$ criteria}

MCI and type of MCI (early MCI or late MCI) will be assessed by the delayed recall score of WMS-III Logical Memory and by the score on the MMSE. On Logical Memory II Story A, a score from 0 to 11 will indicate cognitive impairment per the Alzheimer's Disease Neuroimaging Initiative criteria. MCI type will be determined from this score combined with years of education of the patient. Early MCI (eMCI) will be defined as a delayed recall score of 3-6 with $0-7$ years of education, score of 5-9 with 8-15 years of education and score of 9-11 with 16 or more years of education. Late MCI (IMCI) will be defined as a delayed recall score of $\leq 2$ with $0-7$ years of education, score of $\leq 4$ with $8-15$ years of education and score of $\leq 8$ with 16 or more years of education. For both eMCI and IMCI, everyday function must be well preserved for study inclusion. A MMSE score $\geq 23$ will also be required to indicate mild cognitive impairment, and this is required for study inclusion.

\section{Length of clinical trial}

Most transitions from MCI to AD typically occur within 3 years of follow-up after the diagnosis of MCI is made. ${ }^{34}$ We chose 18 months as the length of this clinical trial to decrease dropouts that can occur in a very long controlled trial. Since this study is considered low risk, we do not anticipate participants to suffer harm from trial participation.

\section{Treatment regimen}

Enrolled participants will come to the clinic for five scheduled visits (weeks $0,12,32,52$ and 78) and will 


\section{Box 1 Inclusion/exclusion criteria}

\section{Inclusion critera}

1. Men and women 55-95 years of age (inclusive) at the time of informed consent.

2. Subjective cognitive complaints, that is, memory or other cognitive complaints, for example, naming/language.

3. Meets criteria for cognitive impairment defined as scores $>1$ SD below standardised norms on memory function as identified by the Wechsler Memory Scale III Logical Memory delayed recall score.

4. Folstein Mini Mental State score $\geq 23$ out of 30 .

5. A family member or other individual who is in contact with the patient and consents to serve as informant during the study; this could be a telephone informant in case of patients who do not have a livein informant or close significant other.

6. Access to a home desktop or laptop computer at acceptable speed for the study duration.

\section{Exclusion criteria}

1. Diagnosis of dementia of any type.

2. Current clinical evidence of schizophrenia, schizoaffective disorder, major depression, psychosis or bipolar I disorder (Diagnostic and Statistical Manual of Mental Disorders IV Text Revision (DSM-IV TR) criteria).

3. Active suicidal ideation or plan.

4. Current or recent (past 6 months) alcohol or substance use disorder (DSM-5 criteria).

5. Clinical stroke with residual neurological deficits. While we will not exclude patients with cerebrovascular disease, we will not include patients who have had a stroke with residual clinical deficits because it is not clear that this type of patient is similar to the $\mathrm{MCl}$ patient generally, and clear-cut neurological impairment, for example, hemiplegia/hemiparesis or speech impairment, may compromise the patient's ability to do the computerised cognitive training or active control procedures and to complete the neuropsychological tests.

6. Use of medications known to have a negative impact on cognition: benzodiazepines in lorazepam equivalents greater than or equal to $1 \mathrm{mg} /$ day, narcotics, anticholinergics. Other patients receive medications that may be associated with cognitive impairment but are rarely considered the likely etiologyaetiology, for example, theophylline, nifedipine, beta blockers; they will not be excluded. Patients receiving other psychotropic medications not expected to have a material impact on cognition, for example, Selective serotonin reuptake inhibitors (SSRIs) and Serotonin-norepinephrine reuptake inhibitors (SNRIs) will be eligible.

7. Presence of any of the following disorders: (a) central nervoussystem infection, with cerebrospinal fluid evidence of meningitis, encephalitis or other infectious process; (b) dementia of any type; (c) Huntington's disease; (d) multiple sclerosis; (e) Parkinson's disease; (f) other neurological disorders with focal signs, for example, amyotrophic lateral sclerosis; $(\mathrm{g})$ mental retardation.

8. Acute, severe unstable medical illness. For cancer, acutely ill patients (including those with metastases) will be excluded, but history of successfully treated cancer will not result in exclusion.

9. Contraindication to MRI scan: pacemaker, metal implants following surgery, any other contraindication to MRI. Eligibility for the MRI scan is a requirement for the study.

10. University of Pennsylvania Smell Identification Test (UPSIT) exclusions: current smoker $>1$ pack daily, current upper respiratory infection (retested as soon as the infection clears). UPSIT scores

Continued
Box 1 Continued

are reduced in schizophrenia, Parkinson's disease and Parkinson'srelated conditions; these disorders are exclusion criteria for this study. Patients with UPSIT exclusions will not receive the UPSIT but will continue to participate in all other aspects of the study.

11. Patients lacking English-speaking ability as determined by self-report and clinical evaluation.

12. Regular online brain training or regular crossword puzzle user, defined as doing these procedures at a frequency of twice weekly or greater during the year prior to screening. Eligible participants who join the trial are instructed not to do these procedures on their own during the trial, that is, independent of the study.

13. Participation in another intervention trial for cognitive impairment.

receive at least three scheduled phone calls with research staff (weeks 20, 42, and 64). Participants will be enrolled into the study after screening for eligibility and consent is signed. The randomisation will be assigned by the statistician and then carried out by the unblinded research coordinator, with individuals stratified by MCI type, age group and site.

\section{Randomisation}

The blinded research coordinator (at NYSPI or Duke) will complete the training group randomisation form to indicate the following information for the patient: site, age, and MCI type. This form will be verified by the unblinded research coordinator at NYSPI, who will then assign a study identification (ID) to that patient, using a prepopulated form from the statistician's randomisation assignment. The order of the study ID assignment will determine which study condition the patient will receive: CPT or CCT. The Lumosity account information will be generated after the MRI has been completed and quality checked. Lumosity account credentials will include a research-specific COGIT ID (cognitive impairment training identification) email address and password, which will enable users to log into an account specific to their study condition.

Randomisation will be complete when the patient logs into his/her account for the first time at the baseline visit and sees which condition he/she is in. At this visit, patients will be trained by unblinded study staff in their assigned training condition. Eighteen modules were selected to target various cognitive domains: (1) memory (tidal treasures, familiar faces, memory matrix); (2) processing speed (speed match); (3) response inhibition (colour match); (4) verbal fluency/vocabulary proficiency (word bubbles, word snatchers, editor's choice, continuum); (5) planning/divided attention (train of thought, brain shift, trouble brewing, disillusion); (6) visual interference (lost in migration, ebb and flow, masterpiece); (7) identification (river ranger); (8) visualisation (speed pack). These cognitive domains were chosen as they are areas that are often impaired in patients with MCI and thus represent areas that can be targeted for improvement. ${ }^{35}$ Verbal fluency and vocabulary proficiency tasks were included 
Table 1 Complete list of CCT game battery and associated cognitive domains (provided by Lumos Labs)

\begin{tabular}{|c|c|}
\hline Game name & Cognitive domain \\
\hline Tidal Treasures & $\begin{array}{l}\text { Working memory } \\
\text { That is, delayed, non-matching to } \\
\text { sample; self-ordered pointing }\end{array}$ \\
\hline Speed Match & Processing speed \\
\hline Colour Match & Response inhibition \\
\hline Word Bubbles & Verbal fluency \\
\hline Train of Thought & $\begin{array}{l}\text { Planning } \\
\text { Divided attention } \\
\text { Multiple attractions }\end{array}$ \\
\hline Familiar Faces & $\begin{array}{l}\text { Episodic memory; verbal memory } \\
\text { and learning }\end{array}$ \\
\hline Memory matrix & $\begin{array}{l}\text { Episodic memory; visuospatial } \\
\text { memory }\end{array}$ \\
\hline Lost in migration & Visual interference \\
\hline Brain shift & Task switching \\
\hline Trouble brewing & $\begin{array}{l}\text { Multitasking, divided attention, } \\
\text { sustained attention, planning, } \\
\text { working memory }\end{array}$ \\
\hline Ebb and flow & $\begin{array}{l}\text { Task switching, semantic and } \\
\text { visual interference }\end{array}$ \\
\hline Masterpiece & $\begin{array}{l}\text { Mental rotation; visualisation; } \\
\text { spatial reasoning }\end{array}$ \\
\hline River ranger & Identification \\
\hline Word Snatchers & Vocabulary proficiency \\
\hline Speed Pack & Visualisation \\
\hline Disillusion & Task switching \\
\hline Editor's choice & Vocabulary proficiency \\
\hline Continuum & Vocabulary proficiency \\
\hline
\end{tabular}

to promote verbal fluency in the CCT group. Further, the episodic memory task, familiar faces, targets verbal memory and learning. With this, it is acknowledged that episodic memory training may be somewhat limited in the selected battery of modules provided by Lumosity. Each CCT session will consist of a random selection of six modules. Participants in the CCT condition are not allowed to choose the games and are not allowed to skip over or change the suite of games. The Lumosity platform will scale difficulty by using the patient's Lumosity Performance Index (LPI). The LPI will consider three areas for each patient. The Game Performance Index (GPI) will be determined by reviewing score distributions for each game. The Cognitive Area Performance Index (speed, memory, attention, flexibility and problem solving) will be calculated using a weighted average of the Game Performance Index. Third, the overall Cognitive Performance Index will be calculated using a weighted average of the GPIs from all cognitive areas. A complete list of selected CCT games are described in table 1. Crosswords engage primarily verbal abilities and perhaps, executive and attentional mechanisms. The Lumosity games target different cognitive domains, such as speed of processing and memory, as well as verbal abilities. The effect that these different trainings have on the so-called far transfer problem will of course be of major interest. There should be no case in which an emergency unblinding will need to take place, as the blinded intervention is a computerised intervention.

Participants in the CCT group and the CPT group will spend the same amount of time on the platform during the intensive training phase, which will consist of four 30-min training sessions per week for 12 weeks. Participants are not required to have any particular level of computer skills for study inclusion; however, at the initial baseline training, all participants will be trained on how to successfully access the training platform and how they could obtain help both from research staff and their informant throughout the study. For both groups, responses will be entered via mouse and keyboard. For the CPT group, questions will not need to be completed in order, and there will not be any feedback for the accuracy of the response at the time of entry by the participant. On completion of the CCT suite of exercises after $30 \mathrm{~min}$, participants will receive a score. Similarly, after $30 \mathrm{~min}$, the crossword training will automatically end. If a participant were to finish an entire crossword puzzle before the $30 \mathrm{~min}$ cut-off, they would be directed to another crossword puzzle to ensure they complete a total of $30 \mathrm{~min}$.

Following the intensive training phase of 12 weeks, participants will be instructed to complete six booster sessions. Each booster session will consist of four CCT/ CPT sessions. Booster sessions will be completed at weeks 20, 32, 42, 52, 64 and 78. At weeks 32, 52 and 78, patients will complete three booster sessions at home and complete the fourth session in-clinic with research staff. At weeks 20, 42 and 64, patients will complete all four booster sessions at home. Generally, in previous cognitive training studies, booster sessions have been limited. For instance, the ACTIVE trial had two booster sessions, each consisting of four 75-min trainings at 11 and 35 months. During the course of the trial, booster sessions included a total of 8 trainings, for a total of 10 hours. $^{6}$ In contrast, COGIT will have 6 booster sessions (24 total training sessions each lasting $30 \mathrm{~min}$ ) over 15 months. Thus, COGIT will include 12 hours total for booster sessions during the trial. The ACTIVE trial only required compliant participants to complete the booster sessions, whereas all participants will complete booster sessions in the COGIT study. ${ }^{6}$

\section{Clinic-based cognitive, functional and smell assessments}

At baseline (week 0), the Alzheimer's Disease Assessment Scale-Cognition Subscale 11 (ADAS-Cog 11) will be administered, in addition to the following neuropsychological test battery: Wechsler Adult Intelligence Scale-III Block Design (to assess visuospatial skills), Digit Symbol Substitution Test (DSST) (to assess attention), Trail Making A \& B (to assess attention and executive function), Verbal Fluency and 15-item Boston Naming Test (to 
assess language), Auditory-Verbal Learning Test (to assess verbal learning and memory) and WMS-III Visual Reproduction Test (to assess nonverbal learning and memory). In addition, the UPSA and University of Pennsylvania Smell Identification Test (UPSIT) will be administered. Testing fatigue is mitigated by allowing participants to take breaks during the testing. If there is missing data from one time point, the study team will attempt to bring the participant back to the clinic within the allowed window to complete missing measures. The FAQ will be administered to the patient's informant, either during the study visit or shortly after the visit over the phone.

\section{Self-Administered cognitive test battery}

Another unique aspect of the study is the use of the NCPT, an online, computerised, self-administered battery developed by Lumosity. It will test various cognitive domains outlined in the Study Measures section. The NCPT will allow us to examine the efficacy of a self-administered test, in combination with standardised, clinic-based neuropsychological tests.

\section{Timeline of longitudinal assessments}

At in-clinic visits (baseline and weeks 12, 52 and 78), the same neuropsychological battery of testing will be completed. At the week 12 and week 78 visits, the patient will be asked to complete the User Engagement Scale, which will be adapted to capture usage of a computerised platform. This scale will measure aspects of engagement, usability and satisfaction with the computerised platform on a 5-point Likert scale. Week 20 will be a phone interview between study physician/neuropsychologist and patient to follow-up on how the patient has been doing and to remind the patient to complete a booster session.

\section{Blinded training procedure}

The blinded research coordinator will administer the full neuropsychological test battery, including the UPSA and FAQ. The blinded clinician will complete the Diagnosis Form and the Contributing Features to MCI form after clinical interview and review of the neuropsychological testing. The unblinded research coordinator will administer the initial computerised training and all subsequent booster sessions to patients in the clinic. To track type of games/crossword puzzles and amount of time that the subject spends doing the games/crossword puzzles, only unblinded study coordinators receive reports from Lumosity each week. If the Lumosity reports of computer games/crosswords access do not match the subject's assigned instructions, the unblinded coordinator then contacts the subject to guide and ensure adherence to the protocol.

\section{Hypotheses}

See figure 1 for a conceptual model of specific study aims and outcome measures. The primary aim of the study is to assess change in cognitive and functional status over 18 months in patients with MCI comparing the CCT and CPT groups. Hypothesis 1: Patients with MCI randomised to CCT will show better cognitive outcomes on the ADAS-Cog 11 (primary outcome measure), Neuropsychological Testing Composite score (secondary outcome measure) and NCPT (exploratory outcome measure) compared with active control (CPT). Hypothesis 2: Patients with MCI randomised to CCT will show better functional outcomes as assessed by the UPSA (primary functional outcome) and FAQ (secondary functional outcome) by the end of the 18-month trial compared with active control. Hypothesis 3: brain pathology (smaller hippocampal volumes, lower odour identification scores on the UPSIT, ApoE e4 allele present) will moderate the relationship between treatment assignment and cognitive and functional outcomes.

The secondary aim of the study is to examine the effects of CCT on resting-state DMN connectivity as well as other networks modulated by CCT effects. Hypothesis 1: MCI patients randomised to CCT will demonstrate greater change in an index of DMN functional connectivity compared with patients randomised to active control. Hypothesis 2: indicators of brain pathology (smaller hippocampal volumes, lower odour identification scores on the UPSIT, ApoE e4 allele present) will moderate the relationship between change in the DMN and treatment assignment.

The tertiary aim of the study is to examine differences in rates of progression to dementia and $\mathrm{AD}$ in the two randomised treatment groups, recognising that if progression to these outcomes is uncommon there will be insufficient statistical power. Hypothesis 1: the proportion converting to dementia will be lower in the CCT group compared with active control.

\section{Study measures}

Study measures with time-points of administration are listed in table 2. The MMSE will be administered at screen and each subsequent in-clinic visit using five different versions of the three-word recall item to reduce practice effects. ${ }^{36}$ The Cumulative Illness Rating Scale for Geriatrics and The Framingham Stroke Risk Scale will be completed by the study physician at screen to assess for cardiovascular disease risk factors and other medical conditions.

The GDS will be administered at screen and each subsequent in-clinic visit to assess for depression. If GDS is greater than five at any visit, the patient will be evaluated by a psychiatrist and an appropriate clinical referral will be made, if needed, for treatment of depression. The Cognitive Reserve Index is a brief questionnaire that will be administered by the research coordinator at screen and will evaluate the cognitive reserve of an individual by means of the compilation of information as it relates to his/her adult life.

At screen, the research coordinator will be responsible for administering the History of Game Use Questionnaire, Physical Activity Assessment and WMS-III Logical Memory I and II. The History of Game Use Questionnaire will be administered again at weeks 12 and 78 to 


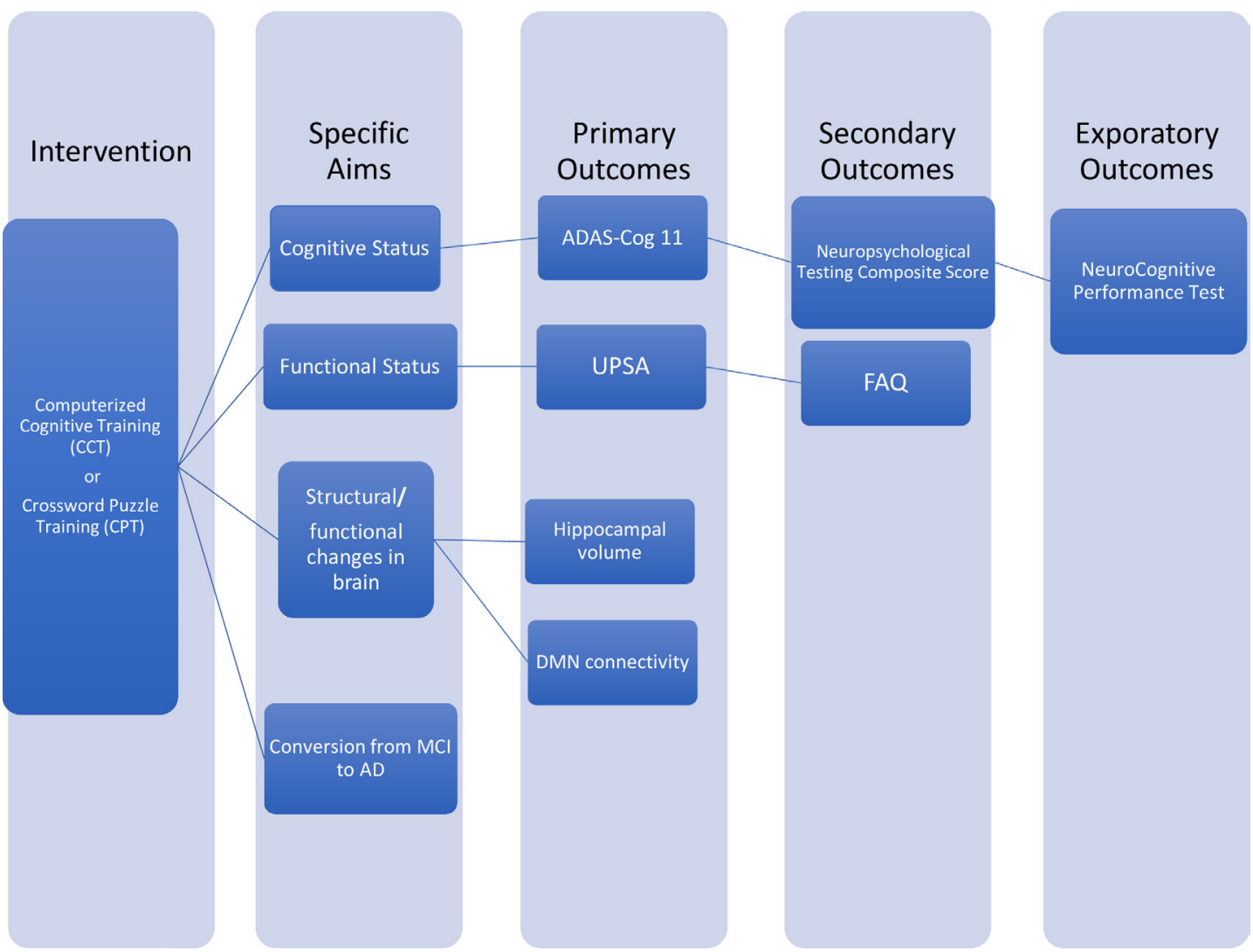

Figure 1 Conceptual model in the intervention phase patients are randomly assigned to either CCT or CPT. To evaluate cognitive status, the primary outcome measure will be the ADAS-Cog 11, the secondary outcome measure will be the neuropsychological testing composite score and the exploratory outcome measure will be the neurocognitive performance test. To evaluate functional status, the primary outcome measure will be the UPSA and the secondary outcome measure will be the FAQ. To evaluate changes in neural circuitry, the primary outcome measures will include hippocampal volume (MRI) and DMN connectivity (fMRI). AD, Alzheimer's disease; ADAS-Cog, 11 Alzheimer's Disease Assessment Scale-Cognitive Subscale 11; CCT, computerised cognitive training; CPT, crossword puzzle training; DMN, default mode network; FAQ, Functional Assessment Questionnaire; fMRI functional MRI; MCI mild cognitive impairment; UPSA UCSD Performance-Based Skills Assessment.

ensure that patients are not partaking in any other types of cognitive training games while in the study.

At screen and week 78, patients will undergo an MRI scan of the brain. The MRI scan will include the following sequences: Localiser, high-resolution T1-weighted Inversion recovery prepped 3DSPGR (spoiled gradient recalled), and T2 FLAIR (fluid attenuated inversion recovery) and GE-EPI (General Electric echo-planar imaging) resting-state fMRI scans.

At weeks 0 and 78, the UPSIT will be completed by the patient, which is a 40 -item scratch and sniff multiple-choice olfactory identification test.

At each in-clinic visit, apart from week 32, the ADAS-Cog 11 and full neuropsychological test battery will be administered. The NCPT will be administered at weeks 0,12 and 78. The cognitive domains measured by the NCPT are memory (visuospatial working memory, short-term memory), processing speed (visual search, psychomotor speed), problem solving (logical reasoning, numerical calculation), attention (selective, divided) and flexibility (response inhibition, task switching). The assessments, 10 total 'subtests', are online adaptations of widely used neuropsychological tests whose test properties are not affected by shifting to computerised administration. ${ }^{37}$

The neuropsychological test battery includes: WAIS-III Block Design, DSST, Trail Making A and B, Verbal Fluency and 15-item Boston Naming Test, Auditory-Verbal Learning Test (AVLT) and WMS-III Visual Reproduction Test. For word learning lists, the neuropsychological testing materials provide different but parallel word lists, so as to avoid practice effects in MMSE and ADAS-Cog, but not for AVLT. With respect to the latter, we did not adopt this approach because we were concerned that different forms have not been established as equivalent in difficulty level. The UPSA will be administered only at weeks 0,32 and 78 due to the high tendency for practice effects. It is a performance-based measure of functional abilities that includes measures of simulated real-world activities; for example, planning a trip to the beach, remembering documents to bring to a medical appointment and dialling a phone number. When a participant wears corrective lenses during the testing battery, this is documented in the participant's research chart. 
Table 2 Table of study procedures

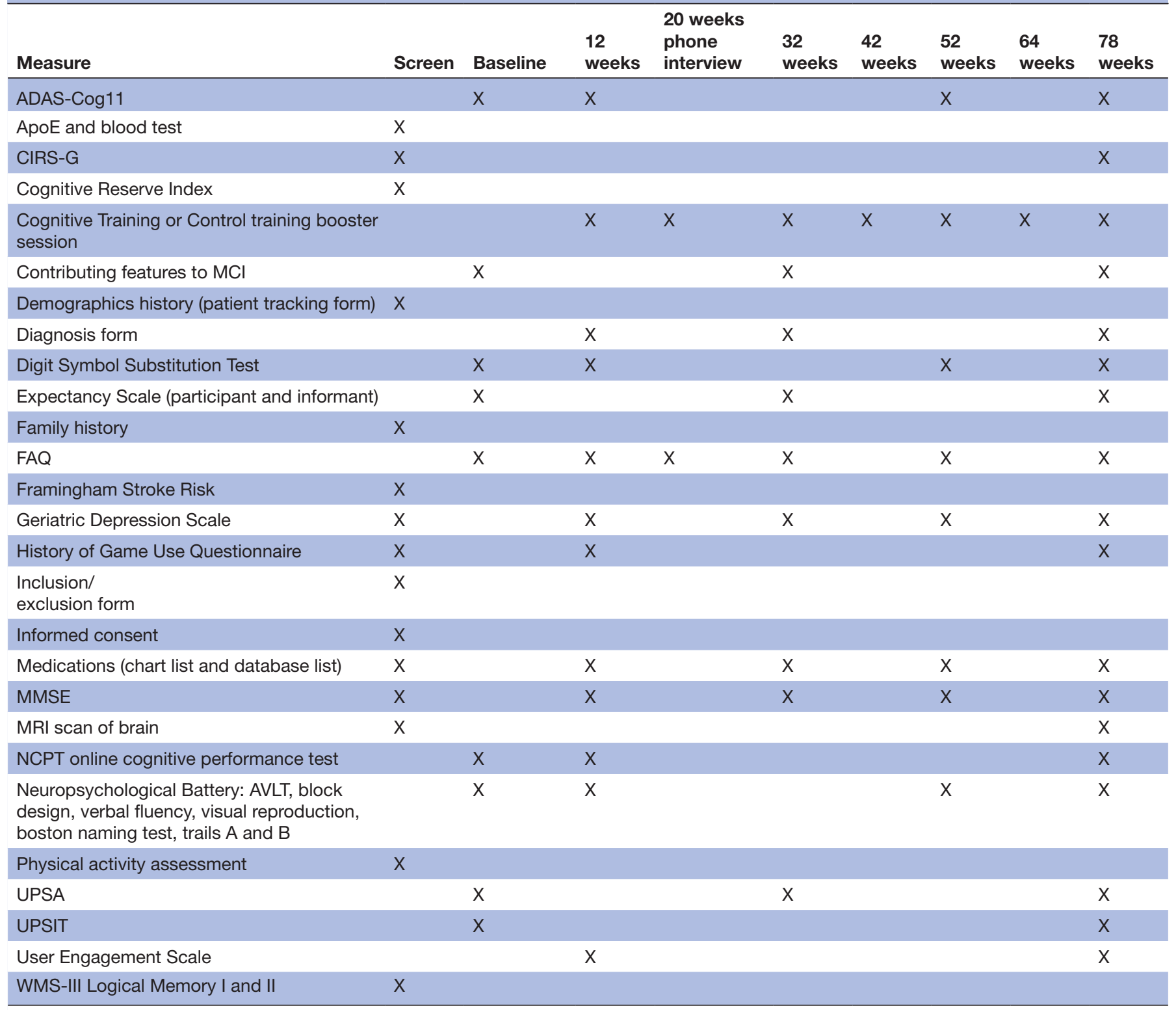

ADAS-Cog 11, Alzheimer's Disease Assessment Scale-Cognitive Subscale 11; AVLT, Auditory Verbal Learning Test; ApoE, Apolipoprotein E gene; CIRS-G, Cumulative Illness Rating Scale for Geriatrics; FAQ, Functional Assessment Questionnaire; MCl, mild cognitive impairment; MMSE, Mini Mental Status Examination; NCPT, NeuroCognitive Performance Test; UPSA, UCSD Performance-Based Skills Assessment; UPSIT, University of Pennsylvania Smell Identification Test; WMS-III, Wechsler Memory Scale-III.

At screen and weeks 12,32, 52 and 78, the participant will meet with the study physician or the neuropsychologist to assess for illness progression and adverse events. Adverse events that are spontaneously reported to research coordinators at any clinic visit will be discussed with the study physician or the neuropsychologist to determine how to proceed. Adverse events and subsequent steps to deal with the adverse events will be documented in the patient chart, and serious adverse events will be reported to the Data Safety and Monitoring Board and study sponsor, National Institute on Aging.

Additionally, the research coordinator will conduct an interview with the informant at, or shortly after, each visit to complete the FAQ.

\section{Criteria for early discontinuation}

We expect early discontinuation to occur because of one or more of the following reasons: (1) the patient's decision not to continue the computerised training (CCT or CPT) due to lack of interest, motivation or available time; (2) unavoidable circumstances, e.g., moving residence and unwillingness to return for in-person evaluations; (3) investigator decision to terminate; (4) death or prolonged hospitalisation for medical reasons. We will not terminate participation for non-adherence because even if the patient is non-adherent to the protocol, we will document level of adherence (done electronically in this computerised training protocol) and still include the 
patient's data in the analyses based on the intent-to-treat principle.

\section{Data management}

Data entry will be completed by programme managers, clinical research coordinators, and research assistants on the study protocol. Data entry/cleaning will be done throughout the project. The data collected in this study will be monitored by the Data Coordinating Center at NYSPI. The unit will work closely with the research assistant/coordinator and the principal investigator to facilitate independent auditing of primary subject records. The database will provide reports indicating all modifications that have been made in the database together with paper communications (fax, e-mail) confirming and authorising these modifications. Access to the data system is available only to authorised users, with multiple levels of security including user ID/password authentication via Microsoft Active Directory overseen by experienced IT personnel. Other authorised users with direct access to the data system will be Data Coordinating Center (DCC) staff. DCC data-related operations and the Scientific Information Retrieval/Citrix system have been certified by Columbia University's Information Security Office. The dataset will not be published in a data repository.

\section{Genetic testing}

Apolipoprotein E (ApoE) genetic analysis on a blood sample will be done through the laboratory of the Human Genetics Resources Core at Columbia University Medical Center. We will assess the ApoE $\varepsilon 4$ allele as potentially associated with response to CCT; a prior trial found an association between the $\varepsilon 4$ allele and cognitive improvement on donepezil. ${ }^{38}$

\section{Concomitant medications}

Putative cognitive enhancers, narcotics, all classes of psychotropic medications and over 20 other classes of commonly prescribed and over-the-counter (and alternative) medications will be documented in a rating form at screen and subsequent in-clinic visits. An exclusion criterion will the be daily use of medications known to have a negative impact on cognition: high-dose narcotics, anticholinergics and benzodiazepines in lorazepam equivalents $\geq 1 \mathrm{mg}$ /day. During the first 12 weeks of the study, the intensive cognitive training phase, patients are encouraged not to change any of their medications, unless clinically indicated.

\section{Statistical analysis and sample size}

We powered our trial to detect an effect size at 18 months of $d=0.58$ ( $80 \%$ power). This effect size is more conservative than published treatment changes associated with CCT (for instance, see reference 39). We assume that dropout is distributed uniformly across waves of follow-up assessments (with 5\% attrition between each consecutive pair of the 5 major time-points, that is, $20 \%$ by 18 months).
Outcome measures (primary and secondary hypotheses testing) Aim 1 hypothesis 1 and 2. Patients with MCI on CCT will show a lower rate of cognitive and functional decline compared with patients with MCI on active control by the end of the 18-month trial. We will use generalised linear mixed effects models of cognitive and functional measures collected repeatedly across the 78 weeks according to the schedule (table 2). For example, cognitive measure ${ }_{\mathrm{ik}}=$ $\beta_{0}+\beta_{1}$ Time $_{\mathrm{ik}}+\beta_{2}$ Group $_{\mathrm{i}}+\beta_{3}\left(\right.$ Group $_{\mathrm{i}} \mathrm{x}$ Time $\left._{\mathrm{ik}}\right)+\mathrm{v}_{0 \mathrm{i}}+$

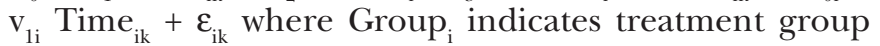
for subject i (group=1 CCT, 0 for control), $k=$ time (baseline, 12 weeks, 20 weeks, 52 weeks, 78 weeks) and $\mathrm{v}_{0 \mathrm{i}}$ is a subject-specific random intercept. Time will be treated as categorical if linearity is not plausible and group effects at 18 months can be tested by forming contrasts from the fitted model. Potential site differences will be evaluated using descriptive statistics, and site will be included in all analyses as a covariate, as will other stratification variables including age group and MCI type at baseline.

Aim 2 hypothesis 1. Patients with MCI randomised to CCT will demonstrate either more of an increase or less of a decrease in DMN connectivity (goodness-of-fit index scores) compared with patients randomised to active control. To test this hypothesis, we will use a repeated measures analysis of covariance (ANCOVA) with time (baseline vs post-treatment) as the repeated measure, DMN connectivity as an outcome, treatment condition (CCT vs CPT) as a predictor, and site, age and MCI status at baseline as covariates.

Moderating effects in aim 1 hypothesis 3 and aim 2 hypothesis 2. As a part of our exploratory analyses, we will examine specific potential moderators: ApoE $\varepsilon 4$ allele, MRI indices, UPSIT. To show, for example, that baseline hippocampal volume is a moderator, we will test its interactive effect with treatment on outcomes. Moderator and moderator-interaction terms can be easily accommodated in the mixed effects regression models described in aim 1. Hypotheses 1 and 2. A similar approach will be used by adding moderator and moderator $\mathrm{x}$ group interactions to the ANCOVA described in aim 2 hypothesis 1 . The results must be interpreted with the caveat that there may not be enough power to assess these interactions, especially for moderators with low prevalence.

Aim 3 hypothesis 1 . The proportion diagnosed with dementia during follow-up will be lower in the CCT group compared with active control. Logistic regression will be used to test the binary outcome of dementia status at 18 months predicted by treatment group controlling for site, age group and MCI type at baseline.

Missing data is managed statistically through use of mixed model repeated measures analyses.

\section{Sample size}

A power analysis was conducted using the RMASS program for longitudinal studies, which determined that a total sample size of 100 participants will provide a sufficient effect size to evaluate our hypotheses. We have two primary outcome measures (ie, multiple outcome measures), 
namely ADAS-Cog and the UPSA. For multiple outcome measures, statistical significance on any one measure is meaningful, and there is no need to correct for multiple comparisons (unlike coprimary outcome measures). All other outcome measures are secondary and exploratory.

\section{Patient and public involvement}

Patients will first be involved in the research after study design is finalised by the study investigators. At this stage, patients will be referred by physicians or self-referred from online and newspaper advertisements for their initial screening visit. The patients will not be involved in study design, study recruitment or conduct, or dissemination of study results. We will assess the burden of the trial intervention on patients using the User Engagement Scale and the Participant/Informant Expectancy Scales. Patients will not be invited to comment on the study design and were not consulted to develop patient relevant outcomes or interpret the results. Patients will not be invited to contribute to the writing or editing of this document for readability or accuracy.

\section{Ethics}

All COGIT patients at entry will be required to have the capacity to provide informed consent and sign the Institutional Review Board (IRB)-approved informed consent form. Local IRB and state regulations for consent will be followed. Patient confidentiality as it pertains to potential and enrolled participants before, during and after the trial will be collected, shared and maintained strictly according to Health Insurance Portability and Accountability (HIPAA) law.

Important protocol modifications will be communicated to the Data Safety and Monitoring Board, NYSPI IRB and Duke University IRB and updated online for trial registries.

The research data on specific moderators, including UPSIT and ApoE genotyping, will not be not released to the patient, and this will be specified in the consent form. The cognitive testing results and clinical reading of the MRI scan will be released to the patient (and the patient's primary physician, if requested); the MRI research volumetric ratings and fMRI findings will not be released.

\section{Data Safety and Monitoring Board}

Three National Institute on Aging-approved independent experts with expertise in conducting clinical trials in mild cognitive impairment will form the Data Safety and Monitoring Board (DSMB). All serious adverse events (SAEs) will be reported to the DSMB. The DSMB will audit the trial conduct, review all SAEs, participate in a teleconference twice a year to determine if the study should continue and then will provide an actionable report to the principal investigator. This process will be independent from the investigators.

\section{Dissemination}

The study results will be disseminated through publications and conference presentations as well as on public websites, including clinicaltrials.gov. Researchers will be eligible for authorship after consideration by the principal investigators; no professional writers will be used.

\section{Significance}

This will be one of the first investigator-blinded and controlled long-term trials to evaluate the effects of home-based CCT versus computerised CPT on cognitive, functional, hippocampal and default mode network connectivity neural outcomes in MCI. Positive results from this pilot trial may support the further development of home-based cognitive training and self-assessments in people at risk for dementia.

The results will help inform the design of a more powerful randomised controlled trial in many ways: determine sample size for a multicenter trial, identify subgroups more likely to benefit, identify subdomains and exercises most likely to improve, optimise training dose and duration, learn how subjects engage, identify gender effects, model slopes and long-term benefits, assess value of a self-administered cognitive test, understand brain networks affected and examine the potential moderating role of ApoE $\varepsilon 4$ status on CCT outcome.

\section{Author affiliations}

${ }^{1}$ Division of Geriatric Psychiatry, New York State Psychiatric Institute, New York, New York, USA

${ }^{2}$ Department of Psychiatry, Columbia University Medical Center, New York, New York, USA

${ }^{3}$ Department of Psychology, Queens College, City University of New York, Flushing, New York, USA

${ }^{4}$ Department of Psychology, The Graduate Center, City University of New York, New York, New York, USA

${ }^{5}$ Department of Biostatistics, Mailman School of Public Health, Columbia University Medical Center, New York, New York, USA

${ }^{6}$ Department of Psychiatry, Duke University Medical Center, Durham, North Carolina, USA

${ }^{7}$ Department of Radiology, Duke University Medical Center, Durham, North Carolina, USA

Contributors DD, MD, JP and JRS conceptualised and designed the study and obtained funding. JD and LSP drafted the initial manuscript. HA contributed to statistics design and JP to design of MRI component. TG, SR, NK, CH, ST, EP assisted with elements of study design, database and conduct. DD is the overall study principal investigator (PI), and MD is the $\mathrm{Pl}$ at the Duke site. All authors (JD, LSP, TG, JRS, SNR, NK, HA, CH, ST, EP, JP, MD, DD) contributed to manuscript edits and revisions and approved the final manuscript as submitted. All authors agree to be accountable for all aspects of the work.

Funding This work is supported by National Institute on Aging, National Institutes of Health, grant number 1R01AG052440-01A1. We thank Lumos Labs for providing the gaming platform at no cost; however, they will not have any involvement in the final design, conduct or analyses of the study.

Competing interests JLD, LSP, TEG, JRS, SNR, NAK, HFA, CAH, SNT, EP, JRP have no competing interests. PMD has received research grants and advisory fees from several companies in this field for other studies, and owns shares in several companies whose products are not discussed here. DPD serves as a consultant on advisory boards to Acadia, Avanir, Genentech, Eisai, and Neuronix.

Patient consent for publication Not required.

Ethics approval This study has been approved by NYSPI IRB, Duke University IRB and Queens College IRB.

Provenance and peer review Not commissioned; externally peer reviewed.

Open access This is an open access article distributed in accordance with the Creative Commons Attribution Non Commercial (CC BY-NC 4.0) license, which 
permits others to distribute, remix, adapt, build upon this work non-commercially, and license their derivative works on different terms, provided the original work is properly cited, appropriate credit is given, any changes made indicated, and the use is non-commercial. See: http://creativecommons.org/licenses/by-nc/4.0/.

\section{REFERENCES}

1. Valenzuela MJ, Sachdev P. Brain reserve and dementia: a systematic review. Psychol Med 2006;36:441-54.

2. Tetlow AM, Edwards JD. Systematic literature review and metaanalysis of commercially available computerized cognitive training among older adults. J Cogn Enhanc 2017;1:559-75.

3. Fisher M, Subramaniam K, Panizzutti R, et al. Computerized cognitive training in schizophrenia: current knowledge and future directions. in cognitive impairment in schizophrenia: characteristics, assessment and treatment. New York, NY: Cambridge University Press; US, 2013: 284-315

4. Preiss M, Shatil E, Čermáková R, et al. Personalized cognitive training in unipolar and bipolar disorder: a study of cognitive functioning. Front Hum Neurosci 2013;7:108.

5. Rapport MD, Orban SA, Kofler MJ, et al. Do programs designed to train working memory, other executive functions, and attention benefit children with ADHD? A meta-analytic review of cognitive, academic, and behavioral outcomes. Clin Psychol Rev 2013;33:1237-52.

6. Rebok GW, Ball K, Guey LT, et al. Ten-Year effects of the advanced cognitive training for independent and vital elderly cognitive training trial on cognition and everyday functioning in older adults. J Am Geriatr Soc 2014;62:16-24.

7. Corbett A, Owen A, Hampshire A, et al. The effect of an online cognitive training package in healthy older adults: an online randomized controlled trial. J Am Med Dir Assoc 2015;16:990-7.

8. Hill NTM, Mowszowski L, Naismith SL, et al. Computerized cognitive training in older adults with mild cognitive impairment or dementia: a systematic review and meta-analysis. AJP 2017;174:329-40.

9. Anguera JA, Boccanfuso J, Rintoul JL, et al. Video game training enhances cognitive control in older adults. Nature 2013;501:97-101.

10. Brehmer Y, Rieckmann A, Bellander M, et al. Neural correlates of training-related working-memory gains in old age. Neuroimage 2011;58:1110-20.

11. Heinzel S, Lorenz RC, Pelz P, et al. Neural correlates of training and transfer effects in working memory in older adults. Neuroimage 2016;134:236-49.

12. Belleville S, Mellah S, de Boysson C, et al. The pattern and loci of training-induced brain changes in healthy older adults are predicted by the nature of the intervention. PLoS One 2014;9:e102710.

13. Kim H, Chey J, Lee S. Effects of multicomponent training of cognitive control on cognitive function and brain activation in older adults. Neurosci Res 2017;124:8-15.

14. Gutchess A. Plasticity of the aging brain: new directions in cognitive neuroscience. Science 2014;346:579-82.

15. Doraiswamy PM. Is the idle mind a devil's workshop? Biol Psychiatry 2012;71:765-6.

16. Engvig A, Fjell AM, Westlye LT, et al. Effects of memory training on cortical thickness in the elderly. Neuroimage 2010;52:1667-76.

17. Petrella JR, Sheldon FC, Prince SE, et al. Default mode network connectivity in stable vs progressive mild cognitive impairment. Neurology 2011;76:511-7.

18. Petrella JR, Prince SE, Wang L, et al. Prognostic value of posteromedial cortex deactivation in mild cognitive impairment. PLoS One 2007;2:e1104.

19. Alvarez Luz Maria, Cortés Sotres JF, León SO, et al. Computer program in the treatment for major depression and cognitive impairment in university students. Comput Human Behav 2008;24:816-26.

20. Elgamal S, McKinnonMC, Ramakrishnan K, et al. Successful computer-assisted cognitive remediation therapy in patients with unipolar depression: a proof of principle study. Psychol Med 2007;37:1229-38.

21. Lohman MC, Rebok GW, Spira AP, et al. Depressive symptoms and memory performance among older adults: results from the active memory training intervention. J Aging Health 2013;25(8 Suppl):209S-29.

22. Owens M, Koster EHW, Derakshan N. Improving attention control in dysphoria through cognitive training: transfer effects on working memory capacity and filtering efficiency. Psychophysiology 2013;50:297-307.

23. Segrave RA, Arnold S, Hoy K, et al. Concurrent cognitive control training augments the antidepressant efficacy of tDCS: a pilot study. Brain Stimul 2014;7:325-31.

24. Bowie CR, Gupta M, Holshausen K, et al. Cognitive remediation for treatment-resistant depression: effects on cognition and functioning and the role of online homework. J Nerv Ment Dis 2013;201:680-5.

25. Naismith SL, Diamond K, Carter PE, et al. Enhancing memory in late-life depression: the effects of a combined psychoeducation and cognitive training program. Am J Geriatr Psychiatry 2011;19:240-8.

26. Basak $\mathrm{C}$, Boot WR, Voss MW, et al. Can training in a real-time strategy video game attenuate cognitive decline in older adults? Psychol Aging 2008;23:765-77.

27. Karbach J, Kray J. How useful is executive control training? age differences in near and far transfer of task-switching training. Dev Sci 2009;12:978-90.

28. Bherer L, Kramer AF, Peterson MS, et al. Transfer effects in task-set cost and Dual-task cost after Dual-task training in older and younger adults: further evidence for cognitive plasticity in attentional control in late adulthood. Exp Aging Res 2008;34:188-219.

29. Ball K, Berch DB, Helmers KF, et al. Advanced cognitive training for $\mathrm{I}$, vital elderly study $\mathrm{G}$. effects of cognitive training interventions with older adults: a randomized controlled trial. JAMA 2002;288:2271-81.

30. De Vriendt $P$, Mets T, Petrovic M, et al. Discriminative power of the advanced activities of daily living (a-ADL) tool in the diagnosis of mild cognitive impairment in an older population. Int Psychogeriatr 2015;27:1419-27.

31. Basak C, Boot WR, Voss MW, et al. Can training in a real-time strategy video game attenuate cognitive decline in older adults? Psychol Aging 2008;23:765-77.

32. Bahar-Fuchs A, Webb S, Bartsch L, et al. Tailored and adaptive computerized cognitive training in older adults at risk for dementia: a randomized controlled trial. J Alzheimers Dis 2017;60:889-911.

33. Herrera C, Chambon C, Michel BF, et al. Positive effects of computer-based cognitive training in adults with mild cognitive impairment. Neuropsychologia 2012;50:1871-81.

34. Petersen RC. Mild cognitive impairment as a diagnostic entity. $J$ Intern Med 2004;256:183-94.

35. Dai W, Lopez OL, Carmichael OT, et al. Mild cognitive impairment and Alzheimer disease: patterns of altered cerebral blood flow at MR imaging. Radiology 2009;250:856-66.

36. Folstein MF, Folstein SE, McHugh PR, et al. A practical method for grading the cognitive state of patients for the clinician. Journal of Psychiatric Research 1975;12:189-98.

37. Morrison GE, Simone CM, Ng NF, et al. Reliability and validity of the neurocognitive performance test, a web-based neuropsychological assessment. Front Psychol 2015;6:1652.

38. Petersen RC, Thomas RG, Grundman M, et al. Vitamin E and donepezil for the treatment of mild cognitive impairment. $N$ Engl J Med Overseas Ed 2005;352:2379-88.

39. Buschert VC, Friese U, Teipel SJ, et al. Effects of a newly developed cognitive intervention in amnestic mild cognitive impairment and mild Alzheimer's disease: a pilot study. J Alzheimers Dis 2011;25:679-94. 
Correction: Cognitive training and neuroplasticity in mild cognitive impairment (COG-IT): protocol for a two-site, blinded, randomised, controlled treatment trial

D'Antonio J, Simon-Pearson L, Goldberg T, et al. Cognitive training and neuroplasticity in mild cognitive impairment (COG-IT): protocol for a two-site, blinded, randomised, controlled treatment trial. BMJ Open 2019;9:e028536. doi: 10.1136/ bmjopen-2018-028536

This article was previously published with an error.

The author name 'Murali Doraiswamy' should be 'P Murali Doraiswamy'.

Open access This is an open access article distributed in accordance with the Creative Commons Attribution Non Commercial (CC BY-NC 4.0) license, which permits others to distribute, remix, adapt, build upon this work non-commercially, and license their derivative works on different terms, provided the original work is properly cited, appropriate credit is given, any changes made indicated, and the use is non-commercial. See:http://creativecommons.org/licenses/by-nc/4.0/.

C C Author(s) (or their employer(s)) 2019. Re-use permitted under CC BY-NC. No commercial re-use. See rights and permissions. Published by BMJ.

BMJ Open 2019;9:e028536corr1. doi:10.1136/bmjopen-2018-028536corr1

D) Check for updates 